\title{
ثنائية اللغة في تعليم اللغة العربية
}

\author{
ريرين وينداساري، \\ جامعة سونان أمبيل الإسلامية الحكومية سورابايا
}

\section{الملخص}

ثنائي اللغة أو ازدواجية اللغة في تعلم اللغة العربية هو أمر غالبًا ما يوجد في إندونيسيا، حتى في جميع البلدان في عملية تعلم اللغات الأجنبية للمتحدثين الأصليين. يهدف استخدام لغتين (ثنائية اللغة) في تعلم اللغة الأجنبية إلى توفير الفهم لمتعلمي اللغة الأجنبية، لأفم اعتادوا على استخدام اللغة الأصلية جنبًا إلى جنب مع أنواع الكلمات وتركيب الجمل، وبالتالي الحاجة إلى تدخلات اللغة الأم للمتحدثين في تعلم اللغات الأجنبية. يختلف مستوى قدرة الطلاب وفقًا لمستواهم، وبالتالي هناك حاجة إلى تفسيرات واضحة من خلال اللغة الأصلية للمتعلم كلغة وسيطة. الكلمات الرئيسية: الثنائية اللغة، اللغة العربية، تعليم

\section{المقدّمة}

يشيع في كثير من بلدان العالم أكثر من لغة رسمية (إمّا لغتان أو أكثر) بمعنى أن الدولة تستعمل لغتين أو أكثر في الأغراض الرسمية، ومن الأمثلة البارزة على ذلك كندا وبلجيكا وسويسرا. وتواجد لغتين أو أكثر في البلد الواحد لايفهم منه أن جميع المواطنين في ذلك البلد يساعملون أو يعرفون أكثر من لغة واحدة، وما يهمنا في هذا الصدد ظاهرة الثنائية اللغوية أو ظاهرة تعدد اللغات في البلد الواحد سواء أكانت هذه اللغات تستخدم للأغراض الرسمية أم خلاف ذلك.

ويقصد بالثنائية اللغوي Bilingualism هي ظاهرة اجتماعية تعني استعمال الفرد أو المجتمع في منطقة معينة للغتين مثل استعمال الفرنسية والألمانية. أكثر من التلامذ يحتاجون إلى مساعدة لغة الأم في تعلّم اللغة الأجنبية مثل 
العربية وكذلك للغة الاجنبية الآخرى. لأفّم يوجد فهم أكبر في يقارن بين اللغة الإندونيسية وللغة العربية. إمّا بدون مساعدة اللغة الاندونيسيا يمكنهم يوجد قليل من الفهم باللغة العربية مع قواعد المختلفة.

بنظر إلى خطوات تعليم اللغة العربية كما ذكر السابق، هذه اسئلة البحث يهلّ عن “ما دور ثنائية اللغة في تعليم اللغة العربية؟ ". وسيلقي الجواب من هذه السؤال في هذه البحث.

دراسة النظرية

أ- الثنائية اللغوية

قال الدكتور محمد علي الخولي في كتابه كانت عدّة تعريف الثنائية اللغوية، منها 1 : أن يتكلم الناس في مجتمع ما لغتين ؛أن يعرف الفرد لغتين. ؛أن يتقن الفرد لغتين ؟أن يستعمل الفرد لغتين. ويلاحظ المرء هذه التعريفات تتسم بالنقص أو الغموض للأسباب الآتية :

1- قد تكون هناك ثنائية لغوية في فرد ما دون أن تكون هذه الثنائية ظاهرة عامة في مجتمع ما. وبعبارة أخرى، إن ما أشار إليه التعريف الأول نوع واحد من أنواع الثنائية، وهو الثنائية المجتمعية. وبذلك فإن التعريف الأول ناقص، حيث إنه لايشير إلى الثنائية الفردية، أي الثنائية المقصورة على الفرد.

2- التعريف الثاني يشير إلى مفهوم (يعرف). ولكن (يعرف) هذه غامضة وواسعة بحيث تعني أشياء غير محددوة. فيما هي درجة هذه المعرفة ؟ وأي المهارات اللغوية معا يجب أن تقع تحت مفهوم (يعرف) ؟ وهل معرفة بعض هذه المهارات دون سواها تكفي لمفهوم الثنائية اللغوية ؟

1 محمد علي الخولي، الحياة مع لغتين (الثنائية اللغوية). (الرياض : جامعة الملك سعود، 1987م) ص. 17 
3- التعريف الثالث يشترط درجة الإتقان في مفهوم الثنائية اللغوية. ولكن ما حكم الملايين من الناس الذين

$$
\text { يعرفون لغتين بدرجات متفاوتة من الإتقان ؟ هل نسميهم أحاديي اللغة؟ }
$$

4- التعريف الرابع يستخدم كلمة (يستعمل). ولكن ما معنى الاستعمال ؟ هل هو الكلام أم القراءة أم الكتابة

أم الاستماع ؟ هل هو المهارات الأربع معا أم واحدة منها أم اثنتان معا أم ثلاث معا ؟ أم مذا ؟

ولفذا يرى البعض أن تعريف الثنائية اللغوية قد يكون عاجزا عن جمع كل أنواعها تحت مظلة واحدة. وما يبرر

هذه الرؤية أيضا هو تنوع حالات الثنائية وظروفها ودرجاتما وأنواعها إلى درجة يصعب معها العثور على تعريف يفي بكل الأغراض والأنواع. ولكن دعونا نجرب هذا التعريف : الثنائية اللغوية هي استعمال الفرد أو الجماعة للغتين بأية درجة من

$$
\begin{aligned}
& \text { درجات الإتقان ولأية مهارة من مهارات اللغة ولأي هدف من الأهداف. } 2 \\
& \text { يرى نبابان فيها عامل ثنائية اللغة في الاندونيسيا، منها33 }
\end{aligned}
$$

1- ي الحديث Sumpah Pemuda سنة 1928، استخدام اللغة الاندونيسيا يتعلق جهاد استقلالية و

$$
\text { مطنية. }
$$

2- اللغات التقاليدية الذي يستخدمها بجموعات سوى اللغة الاندونيسيا في العاد و في انشطة اليومية.

$$
\text { 3- متزوجة المخلطة بين أصحاب التقليدية المختلفة. }
$$

4- هجرة المجتمع من ولاية إلى ولاية أخرى مثل تمدّن و ترنسميكراسي و انتقال العمّال و غير ذلك.

5- اتّصال بين القابلة المختلفة من جانب بتحارية و اجتماعية و عمل الحكومية.

$$
\text { 6- - كثرة دافع من حيث احتياجات المهنية و المعيشة. }
$$

$$
\text { 2محمد علي الحولي، الحياة مع لغتين (الثنائية اللغوية). (الرياض : جامعة الملك سعود، 1987م) ص. } 18
$$

3

Rohmani Nur Indah dan Abdurrahman, Psikolinguistik: Konsep dan Isu Umum. (Malang : UIN Malang Press 2008) Hal. 72 
يجب الانتباه إلى فرق هام بين الثنائية الفردية Individual Bilingualism والثنائية الاجتماعية Societal B Bilingualism ألاوهو أن الثنائية الفردية تشير إلى أن الفرد نفسه يستعمل لغتين. ولكن الثنائية اللغة. بل تعني أن معظم أفراد ذلك المجتمع ثنائيّو اللغة كي يتمكنوا من التفاهم في حياهم اليومية. والثنائية المجتمعية ثلاثة أنماط 4: ثنائية أفقية Horizontal Bilingualism : وهي أن تكون اللغتان متساويتين في المكانة رسميا وثقافيا واجتماعيا. مثال ذلك الإنجليزية والفرنسية في مقاطعة كويبك في كندا.

ثنائية رأسية Vertical Bilingualism : أن تكون لهجة فصيحة وأخرى عامية. مثال ذلك الفصيحة والعامية في البلاد العربية. وهذه الحالة في الواقع ثنائية لهجية. ويدعوها البعض ازدواجية لغوية.

ثنائية قطرية Diagonal Bilingualism: أن يستخدم الجمتمع لغة فصيحة ولهجة عامية من لغة أخرى. مثال ذلك الإنجليزية زالفرنسية العامية في لويزيانا في الولايات المتحدة الأمريكية. 5

\section{أ) أثنائية اللغوية وتوزيع الاستعمال}

في بعض الحالات يستخدم الناس اللغتين تبادليا، أي من الممكن أن يستخدم المرء اللغة الأولى أو اللغة الثانية في

أي مكان وزمان. وتدعى هذه الثنائية ثنائية تبادلية Reciprocal Bilingualism. ولكن في بعض الحالات. نجد أنّ Complementary الفرد يستخدم اللغتين تكامليا، أي يخصص لكل لغة أدوارا معينة. وتدعى هذه الحالة ثنائية تكاملية : Bilingua lism

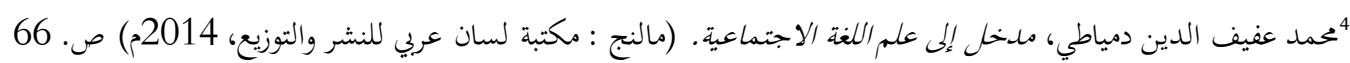

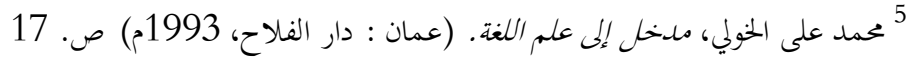

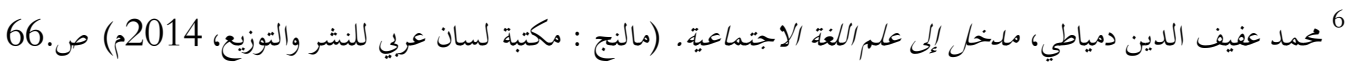


1 - تكاملية مكانية. يقوم الفرد باستخدام اللغة الأولى في البيت واللغة الثانية خارجه، أويستخدم اللغة الأولى في

$$
\text { العمل واللغة الثانية خارجه. }
$$

2- تكاملية موضوعية. يقوم الفرد باستخدام اللغة الأولى في الحديث عن موضوعات معينة واللغة الثانية في الحديث عن موضوعات أخرى. مثلا إذا تحدث عن الأمور اليومية استخدم اللغة الولى، وإذا تحدث عن

\section{أمور عملية استخدم اللغة الثانية.}

3- تكاملية بشرية. يقوم الفرد هنا باستخدام اللغة الأولى مع عدد معين من الناس واللغة الثانية مع سواهم، كأن

$$
\text { يستخدم اللغة الأولى مع أهل بيته واللغة الثانية مع سواهم. }
$$

ويجب ألا ننسى حالة شائعة لدينا جميعا من الثنائية اللغوية الرأسية والتي يمكن أن ندعوها الثنائية اللهجية bidialectalism للهجتين من لغة واحدة وبصورة تكاملية. ففي حالة المثقفين العرب، تراهم يستخدمون العربية الفصحية في التعليم والمحاضرات والخطب الرسمية والكتابة ونشرات الأخبار، ويستخدمون العامية في البيت الشارع. 8

وإذا عدنا قليلا لتفضيل استخدام مصطلح الثنائية اللغوية على الازدواجية اللغوية، نجد أن أحد اسباب هذا التفضيل هو أن مصطلح الازدواجية اللغوية اشتهر عنه يرادف مصطلح الثنائية اللهجية. ولذا كما ذكرت في المبحث الأول من هذا الفصل آثرت مصطلح الثنائية اللغوية على مصطلح الازدواجية أو اللغة. ويمكن للمرء أن يلاحظ أن التوزيع التكاملي في الثنائية التكاملية يمكن أن يتخذ أحد الأنماط الآتية 9.

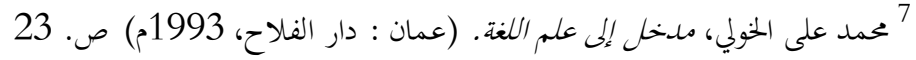

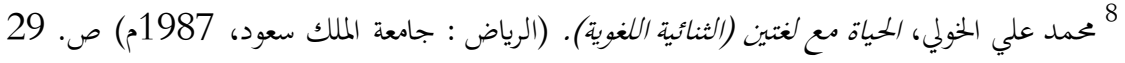

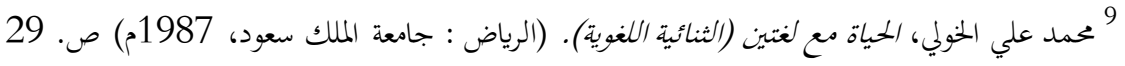


1- التوزيع المكاني : في هذه الحالة، يقوم الفرد بتخصيص مكان واحد أو أكثر لكل لغة من اللغتين. كأن يخصص ل1 للتفاهم داخل البيت و ل2 للتفاهم خارجه.

2- التوزيع الموضوعي : هنا يخصص الفرد لكل لغة موضوعات معينة. فإذا تحدث مثلا في شؤون الحياة العادية، استعمال ل1، وإذا تحدث في التكنولوجيا استعمال ل2. إذا تحدث في الفن والأدب والشعر استخدام ل1، وإذا تحدث في علم الإحصاء استخدام ل2. وبعبارة أخرى، يتم التوزيع هنا على أساس طبيعة الموضوع.

3- التوزيع البشري : هنا يخصص الفرد لغة معينة للتفتاهم مع فئة محددة من الناس. كأن يخصص ل 1 للتحدث مع زوجته و أبنائه ووالديه ويخصص ل2 للتحدث مع من سواهم من الناس. وقد يخصص ل 1 للتفاهم مع أفراد بجموعة معينة من الناس ويخصص ل2 للتفاهم مع أفراد مجموعة أخرى.

\section{ب- ثائية اللغة في تعليم اللغة العربية}

إن اللغة العربية لدى الطلاب غير العرب تعتبر لغة أجنبية يختلف تعليم وتعلّمها عند الطلبة العرب. فتعليم اللغة الأجنبية هو نشاط مقصود يقوم فرد ما لمساعدة فرد آخر على الاصال بنظام من الرموز اللغوية يختلف عن ذلك الذي ألفه وتوّد الاتصال به. 10 وتعليم اللغة العربية كثير يعقد بالثنائية اللغوية لأنّ في التعليم والتعلم الثنائي اللغة تظهر

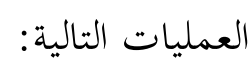

-1 - - مبادئ تعليم ثنائي اللغة

هناك بجموعة من المبادئ الخاصة بالتعليم ثنائي اللغة بحيث يمكن للمهتمين التركيز عليها وفهمها وهي الطالب يعد عنصرا نشطا في اكتساب اللغة.6يتم اكتساب اللغات، وليس دراستها،بناء الطلاب قواعد خاصة بهم في اكتساب اللغة. 10 شدي احمد طعيمة, تعليم اللغة العببية لغير الناطقين كما ـ (مصر : جامعة المنصورة، 1989م )ص. 110 


$$
\begin{aligned}
& \text { 2- اكتساب اللغة في التعليم الثنائي اللغة } \\
& \text { أ) نظريات اكتساب اللغة }
\end{aligned}
$$

إن في اكتساب اللغة نظريات قدمها العلماء اللغويون، وفي ـذا البحث ينحصر تقديدها على ثلاثة

$$
\text { نظريات. }
$$

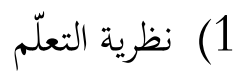

وأنّ اللغة يتم اكتساها عن طريقة التقليد والمحاكاة من الطفل لألفاظ الكبار من نطق الحروف

والكلمات والجمل. وذلك لأن اللغو يتم تعلمها بنفس الطريقة التي نتعلم بها أنواع السلوك

$$
\text { الأخرى. }
$$

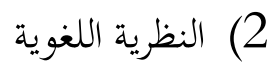

يرى تشومسكي أن كل طفل يمتلك قدرة فطرية تمّكنه من اكتساب اللغة على أساس وجود

$$
\text { نماذج أولية للصياغة اللغوية لدى الأطفال. }
$$

$$
\text { 3) النظرية المعرفية }
$$

إن الكفاءة لاتكتسب إلا بناء على تنظيمات داخلية تبدأ أولية ثم يعاد تنظيما وفق تفاعل

$$
\begin{aligned}
& \text { الطفل مع البيئة الخارجية. } \\
& \text { 3- - فهم المواد في التعليم الثنائي اللغة }
\end{aligned}
$$

إن الدعلومات التي سيحصل عليها الطلاب من الدواد الدراسية إما أن يقدمها الدعلم عن طريق

المحاضرة وإما أن ينالوها عن طريق القراءة في النصوص في الكتب الدراسية. ويحتاج الطلاب في فهم تلك

الدعلومات خبرا سماعيا كان أو نصا كتابيا إلى طرق ومهارات خاصة لكي يفهموها فهما صحيحا. 11 
وفي تعليم اللغة العربية لغير الناطقين بها لازم أن يستخدم ثنائية اللغة لفهم الطلاب معنى الكلمات في هذه اللغة. هناك أنواع طريقة في تعليم اللغة العربية مثل طريقة قواعد والترجمة و غير ذلك من الطرق التعليم. تجعل هذه الطريقة هدفها الأول تدريس قواعد اللغة الأجنبية، ودفع الطالب إلى حفظها واسظهارها، ويتم تعليم اللغة عن طريقة الترجمة بين اللغتين : الأم والاجنبية، وتَتم هذه الطريقة بتنمية مهارتي القراءة والكتابة في اللغة الأجنبية. تستخدم هذه الطريقة اللغة الأم للمتعلم كو سيلة رئيسية لتمعليم اللغة المنشودة. 12

الخملاصة

هناك حاجة إلى ثنائية اللغة في تعلم اللغة العربية للطلاب بخلاف اللغة العربية واللغة الاندونسيا. لأفّمم معتادون على اللغة الاندونيسيا لفهم و لشرح اللغة العربية، قبل تطبيق اللغة العربية حول أنشطة اليومية. فيها أكثر خلافية و متساوية في حال قواعد بين اللغة العربية و اللغة الاندونسيا، لذا يريد طلاب أن يفهم قواعد اللغة العربية بواسطة قواعد اللغة الاندونسيا.

لكن هذه الحال غير مناسب في مرحلة العالية، وأمّا في مرحلة العالية يحتاجون الطلاب كثير ممارسة في حال أربع مهارات كلّه متكامل غير متفرّقة، لأهّم قد ينالو أكثر مفردات بصيغ متنوّعة. فأّما في حال الابتدائية، إذا مباشرة يشرح المعلّم بالعربية، فهم يفهمون بالاشارة فقط، والآخير هم يحتاجون إلى توضيح باللغة الاندونيسيا أيضا و كذالك في مرحلة الثانوية.

إنّ استخدام ثنائية اللغة في تعليم اللغة الأجنبية مثل العربية مشهور حول متعلّم اللغة العربية بفضل العلم الدينية مثل الفقه و العقيدة و التصوّف عند في المعاهد الاسلامية. فبذلك ينبغي على طلاب المعهد يتعلّمون اللغة العربية لفهم هاؤلاء الدرس. أمّا طريقة التي تستخدم في أمثر المعاهد الاسلامية هي طريقة و قواعد والترجمة. منها تتنوّن من قراءة النص 12 محمد طاهر و الأصدقاء، المدخل إلى طرق تدريس العربية للاندونسييين. (سورابايا : سونن أمبيل بريس، 2013م) ص. 50 
،و عناصر القواعد لكله الكلمة، وشرح الكلمة في جملة الفيدة، ومعنه من حيث دلالة الألفاظ بها. هذه طريقة متكاملة للمبتدئين اللغة العربية، و تطبيقها تحسين كقاءة العربية لدى الطلاب من حيث قولعد اللغة العربية.

فلأن تعلّم اللغة العربية الآن تحتاج إلى كثير تطبيق اللغة من حيث البيئة مناسب بهدف تعلّم اللغة العربية. ثنائية اللغة مساعد في عملية التعليم ولكن لم يكثر عند تطبيقها. و استخدام اللغة الوسيطة لتشريح الصعوبة فقط. المراجع محمد علي الخولي. 1987. الحياة مع لغتين (الثنائية اللغوية). الرياض : جامعة الملك سعود. محمد على الخولي. 1993. مدخل إلى علم اللغة. عمان : دار الفلاح. رشدي احمد طعيمة. 1989. تعليم اللغة العربية لغير الناطقين كما ـمصر : جامعة المنصورة. محمد عفيف الدين دمياطي. 2013. مداخل إلى علم اللغة الاجتماعية .مالنج : مكتبة لسان عربي للنشر والتوزيع. محمد طاهر و الأصدقاء. 2013. المدخل إلى طرق تلدريس العببية للاندونسييين. سورابايا : سونن أمبيل بريس. Indah, Rohmani Nur dan Abdurrahman.2018. Psikolinguistik: Konsep dan Isu Umum. Malang : UIN Malang Press. 